\title{
Complex manifolds with split tangent bundle
}

\author{
Arnaud Beauville ${ }^{1}$
}

\section{Introduction}

The theme of this note is to investigate when the tangent bundle of a compact complex manifold $\mathrm{X}$ splits as a direct sum of sub-bundles. This occurs typically when the universal covering space $\widetilde{\mathrm{X}}$ of $\mathrm{X}$ splits as a product $\prod_{i \in \mathrm{I}} \mathrm{U}_{i}$ of manifolds on which the group $\pi_{1}(\mathrm{X})$ acts diagonally (that is, $\pi_{1}(\mathrm{X})$ acts on each $\mathrm{U}_{i}$ and its action on $\widetilde{\mathrm{X}}=\prod \mathrm{U}_{i}$ is the diagonal action $\left.g \cdot\left(u_{i}\right)=\left(g u_{i}\right)\right)$ : the vector bundles ${ }^{2}$ $\mathrm{T}_{\mathrm{U}_{i}}$ on $\widetilde{\mathrm{X}}$ are stable under $\pi_{1}(\mathrm{X})$, hence the decomposition $\mathrm{T}_{\widetilde{\mathrm{X}}}=\oplus_{i} \mathrm{~T}_{\mathrm{U}_{i}}$ descends to a direct sum decomposition of $\mathrm{T}_{\mathrm{X}}$. For Kähler manifolds, it is tempting to conjecture that the converse is true, namely that any direct sum decomposition of the tangent bundle $\mathrm{T}_{\mathrm{X}}$ (perhaps with the additional assumption that the direct summands are integrable) gives rise to a splitting of the universal covering. We will show that this is indeed the case in three different situations:

a) X admits a Kähler-Einstein metric;

b) $\mathrm{T}_{\mathrm{X}}$ is a direct sum of line bundles of negative degree;

c) $\mathrm{X}$ is a Kähler surface.

Case $a$ ) is a direct consequence of the fact that on a compact Kähler-Einstein manifold, any endomorphism of the tangent bundle is parallel (this idea appears for instance in $[\mathrm{Y}]$, and in a more implicit form in $[\mathrm{K}]$ ). Case $b$ ) is a slight improvement of a uniformization result of Simpson [S]. To treat case $c$ ) we use the classification of surfaces and some simple remarks about connections. The result in this case is actually an easy consequence of the paper $[\mathrm{K}-\mathrm{O}]$, where the authors classify surfaces with a holomorphic conformal structure - this turns out to be closely related to the question we are studying here. However we found simpler and more enlightening to give an independent proof rather than extracting from $[\mathrm{K}-\mathrm{O}]$ the pieces of information that we need.

In $\S 2$ we give a few examples which show that for non-Kähler manifolds a splitting of the tangent bundle does not necessarily imply a splitting of the universal covering.

1 Partially supported by the European HCM project "Algebraic Geometry in Europe" (AGE).

2 Throughout the paper we will abuse notation and write $\mathrm{T}_{\mathrm{U}_{i}}$ instead of $p r_{i}^{*} \mathrm{~T}_{\mathrm{U}_{i}}$. 


\section{Kähler-Einstein manifolds}

Theorem A - - Let X be a compact complex manifold admitting a Kähler-Einstein metric. Assume that the tangent bundle of $\mathrm{X}$ has a decomposition $\mathrm{T}_{\mathrm{X}}=\underset{i \in \mathrm{I}}{\oplus} \mathrm{E}_{i}$. Then the universal covering space of $\mathrm{X}$ is a product $\prod_{i \in \mathrm{I}} \mathrm{U}_{i}$ of complex manifolds, in such a way that the decomposition $\mathrm{T}_{\mathrm{X}}=\underset{i \in \mathrm{I}}{\oplus} \mathrm{E}_{i}$ lifts to the decomposition $\mathrm{T}_{\Pi \mathrm{U}_{i}}=\underset{i \in \mathrm{I}}{\oplus} \mathrm{T}_{\mathrm{U}_{i}}$; the group $\pi_{1}(\mathrm{X})$ acts diagonally on $\prod_{i \in \mathrm{I}} \mathrm{U}_{i}$.

The proof follows closely that of thm. 2.1 in [Y] (I am indebted to J. Wahl for pointing out this reference).

Proof: (1.1) As a consequence of the Bochner formula, every endomorphism of $\mathrm{T}_{\mathrm{X}}$ is parallel $[\mathrm{K}]$. This applies in particular to the projectors associated to the direct sum decomposition of $\mathrm{T}_{\mathrm{M}}$; therefore the sub-bundles $\mathrm{E}_{i}$ are preserved by the hermitian connection, hence the holonomy representation of $\mathrm{X}$ is the direct sum of a family of representations corresponding to the $\mathrm{E}_{i}$ 's. By the De Rham theorem, the universal covering space of $\mathrm{X}$ splits as a product $\prod_{i \in \mathrm{I}} \mathrm{U}_{i}$, such that the decomposition $\mathrm{T}_{\mathrm{X}}=\oplus_{i \in \mathrm{I}} \mathrm{E}_{i}$ pulls back to the decomposition $\mathrm{T}_{\Pi_{\mathrm{U}}}=\underset{i \in \mathrm{I}}{\oplus} \mathrm{T}_{\mathrm{U}_{i}}$.

(1.2) The last assertion follows from the following simple observation: if $a$ group $\Gamma$ acting on a product $\prod_{i \in \mathrm{I}} \mathrm{U}_{i}$ preserves the decomposition $\mathrm{T}_{\Pi_{\mathrm{U}_{i}}}=\underset{i \in \mathrm{I}}{\oplus} \mathrm{T}_{\mathrm{U}_{i}}$, it acts diagonally. Let indeed $\gamma$ be an automorphism of $\prod_{\mathrm{U}_{i}}$; for $j \in \mathrm{I}$, put $\gamma_{j}=p r_{j} \circ \gamma$. The condition $\gamma^{*} \mathrm{~T}_{\mathrm{U}_{j}}=\mathrm{T}_{\mathrm{U}_{j}}$ means that the partial derivatives of $\gamma_{j}$ in the directions of $\mathrm{U}_{k}$ for $k \neq j$ vanish, hence $\gamma_{j}\left(\left(u_{i}\right)_{i \in \mathrm{I}}\right)$ depends only on $u_{j}$, which gives our claim.

\section{Non-Kähler examples}

In this section we give examples of manifolds for which the tangent bundle is a direct sum of line bundles, but which do not satisfy the conclusions of Theorem A. (2.1) Hopf manifolds

Let $\mathrm{T}=\operatorname{diag}\left(\alpha_{1}, \ldots, \alpha_{n}\right)$ be a diagonal matrix, with $0<\left|\alpha_{i}\right|<1$ for each $i$. The cyclic group $T^{\mathbf{Z}}$ generated by $T$ acts freely and properly on $\mathbf{C}^{n}-\{0\}$; the quotient $\mathrm{X}$ is a compact complex manifold, called a Hopf manifold. For each non-zero complex number $\theta$, denote by $\mathrm{L}_{\theta}$ the flat line bundle associated to the character of $\pi_{1}(\mathrm{X})=\mathrm{T}^{\mathbf{Z}}$ mapping $\mathrm{T}$ to $\theta$; in other words, $\mathrm{L}_{\theta}$ is the quotient of the trivial line bundle $\left(\mathbf{C}^{n}-\{0\}\right) \times \mathbf{C}$ by the action of the automorphism $(\mathrm{T}, \theta)$. By construction we have $\mathrm{T}_{\mathrm{X}}=\bigoplus_{i=1}^{n} \mathrm{~L}_{\alpha_{i}}$, but the universal covering space $\mathbf{C}^{n}-\{0\}$ of $\mathrm{X}$ is clearly not a product.

(2.2) Complex compact nilmanifolds

These are compact manifolds $\mathrm{X}=\mathrm{G} / \Gamma$, where $\mathrm{G}$ is a nilpotent complex Lie 
group and $\Gamma$ a discrete subgroup of $G$. We may assume that $G$ is simply-connected and non-commutative (to exclude the trivial case of complex tori). A well-known example is the Iwasawa manifold $\mathrm{U}(\mathbf{C}) / \mathrm{U}(\mathbf{Z}[i])$, where $\mathrm{U}$ is the group of uppertriangular $3 \times 3$ matrices with diagonal entries 1 ; many examples can be obtained in an analogous way.

The tangent bundle of $X=G / \Gamma$ is trivial, and its universal covering space $\mathrm{G}$ is isomorphic to $\mathbf{C}^{n}$; however we claim that whatever isomorphism $\mathrm{G} \stackrel{\sim}{\longrightarrow} \mathbf{C}^{n}$ we choose, the action of $\Gamma$ cannot be diagonal. Indeed if $\Gamma$ acts diagonally, the standard trivialization of $\mathrm{T}_{\mathbf{C}^{n}}$ deduced from the coordinate system descends to a trivialization of $\mathrm{T}_{\mathrm{X}}$. Any such trivialization lifts to a trivialization of $\mathrm{T}_{\mathrm{G}}$ defined by a basis of right invariant vector fields; therefore the standard trivialization of $\mathrm{T}_{\mathbf{C}^{n}}$ is G-equivariant. In view of 1.2 this means that $\mathrm{G}$ itself acts diagonally on $\mathbf{C}^{n}$, hence $\mathrm{G}$ embeds into $\operatorname{Aut}(\mathbf{C})^{n}$. Now any nilpotent connected subgroup of the affine group $\operatorname{Aut}(\mathbf{C})$ is commutative, so we conclude that $G$ is commutative, contrary to our hypothesis.

\section{Simpson's uniformization result}

The following lemma, which is a variation on the Baum-Bott theorem [B-B], will allow us to slightly improve Simpson's result:

Lemma 3.1.- Let $\mathrm{X}$ be a complex manifold, and $\mathrm{E}$ a direct summand of $\mathrm{T}_{\mathrm{X}}$. The Atiyah class at $(\mathrm{E}) \in \mathrm{H}^{1}\left(\mathrm{X}, \Omega_{\mathrm{X}}^{1} \otimes \mathcal{E} n d(\mathrm{E})\right)$ comes from $\mathrm{H}^{1}\left(\mathrm{X}, \mathrm{E}^{*} \otimes \mathcal{E} n d(\mathrm{E})\right)$. In particular, any class in $\mathrm{H}^{r}\left(\mathrm{X}, \Omega_{\mathrm{X}}^{r}\right)$ given by a polynomial in the Chern classes of E vanishes for $r>\operatorname{rk}(\mathrm{E})$.

Proof: Write $\mathrm{T}_{\mathrm{X}}=\mathrm{E} \oplus \mathrm{F}$; let $p: \mathrm{T}_{\mathrm{X}} \rightarrow \mathrm{E}$ be the corresponding projection. For any sections $\mathrm{U}$ of $\mathrm{E}$ and $\mathrm{V}$ of $\mathrm{F}$ over some open subset of $\mathrm{X}$, put $\mathrm{D}_{\mathrm{V}} \mathrm{U}=$ $p([\mathrm{~V}, \mathrm{U}])$. This expression is $\mathcal{O}_{\mathrm{X}}$-linear in $\mathrm{V}$ and satisfies the Leibnitz rule $\mathrm{D}_{\mathrm{V}}(f \mathrm{U})=f \mathrm{D}_{\mathrm{V}}(\mathrm{U})+(\mathrm{V} f) \mathrm{U}$, so that $\mathrm{D}$ is a $\mathrm{F}$-connection on $\mathrm{E}[\mathrm{B}-\mathrm{B}]$ : if we denote by $\mathcal{D}^{1}(\mathrm{E})$ the sheaf of differential operators $\Delta: \mathrm{E} \rightarrow \mathrm{E}$, of degree $\leq 1$, whose symbol $\sigma(\Delta)$ is scalar, this means that $\mathrm{D}$ defines an $\mathcal{O}_{\mathrm{X}}$-linear map $\mathrm{F} \rightarrow \mathcal{D}^{1}(\mathrm{E})$ such that $\sigma\left(\mathrm{D}_{\mathrm{V}}\right)=\mathrm{V}$ for all local sections $\mathrm{V}$ of $\mathrm{F}$. Thus the exact sequence

$$
0 \rightarrow \mathcal{E} n d(\mathrm{E}) \longrightarrow \mathcal{D}^{1}(\mathrm{E}) \stackrel{\sigma}{\longrightarrow} \mathrm{T}_{\mathrm{X}} \rightarrow 0
$$

splits over the sub-bundle $\mathrm{F} \subset \mathrm{T}_{\mathrm{X}}$; therefore its extension class at $(\mathrm{E}) \in$ $\mathrm{H}^{1}\left(\mathrm{X}, \Omega_{\mathrm{X}}^{1} \otimes \mathcal{E} n d(\mathrm{E})\right) \quad$ vanishes $\quad$ in $\mathrm{H}^{1}\left(\mathrm{X}, \mathrm{F}^{*} \otimes \mathcal{E} n d(\mathrm{E})\right)$, hence comes from $\mathrm{H}^{1}\left(\mathrm{X}, \mathrm{E}^{*} \otimes \mathcal{E} n d(\mathrm{E})\right)$. The last assertion follows from the definition of the Chern classes in terms of the Atiyah class.

We denote as usual by $\mathbf{H}$ the Poincaré upper half-space. 
Theorem B.- Let X be a compact Kähler manifold, with a Kähler class $\omega$. Assume that the tangent bundle $\mathrm{T}_{\mathrm{X}}$ is a direct sum of line bundles $\mathrm{L}_{1}, \ldots, \mathrm{L}_{n}$ with $\omega^{n-1} \cdot c_{1}\left(\mathrm{~L}_{i}\right)<0$ for each $i$. Then the universal covering space of $\mathrm{X}$ is $\mathbf{H}^{n}$, and the decomposition $\mathrm{T}_{\mathrm{X}}=\oplus \mathrm{L}_{i}$ lifts to the canonical decomposition $\mathrm{T}_{\mathbf{H}^{n}}=\left(\mathrm{T}_{\mathbf{H}}\right)^{\oplus n}$. Proof: This is Cor. 9.7 of $[\mathrm{S}]$, except that Simpson makes the extra hypothesis $\omega^{n-2} \cdot\left(c_{1}(\mathrm{X})^{2}-2 c_{2}(\mathrm{X})\right)=0$ (the assertion about the compatibility of decompositions is not stated in loc. cit., but follows directly from the proof). Now lemma 3.1 gives $c_{1}\left(\mathrm{~L}_{i}\right)^{2}=0$ for each $i$, hence $c_{1}(\mathrm{X})^{2}-2 c_{2}(\mathrm{X})=0$.

\section{The surface case}

Theorem C.- Let $\mathrm{X}$ be a compact complex surface. The tangent bundle of $\mathrm{X}$ splits as a direct sum of two line bundles if and only if one of the following occurs:

a) The universal covering space of $\mathrm{X}$ is a product $\mathrm{U} \times \mathrm{V}$ of two (simplyconnected) Riemann surfaces and the group $\pi_{1}(\mathrm{X})$ acts diagonally on $\mathrm{U} \times \mathrm{V}$; in that case the given splitting of $\mathrm{T}_{\mathrm{X}}$ lifts to the direct sum decomposition $\mathrm{T}_{\mathrm{U} \times \mathrm{V}}=\mathrm{T}_{\mathrm{U}} \oplus \mathrm{T}_{\mathrm{V}}$.

b) $\mathrm{X}$ is a Hopf surface, with universal covering space $\mathbf{C}^{2}-\{0\}$. Its fundamental group is isomorphic to $\mathbf{Z} \oplus \mathbf{Z} / m \mathbf{Z}$, for some integer $m \geq 1$; it is generated by a diagonal automorphism $(x, y) \mapsto(\alpha x, \beta y)$ with $|\alpha| \leq|\beta|<1$, and a diagonal automorphism $(x, y) \mapsto(\lambda x, \mu y)$ where $\lambda$ and $\mu$ are primitive $m$-th roots of 1 .

As a corollary, for Kähler surfaces we see that any direct sum decomposition of the tangent bundle gives rise to a splitting of the universal covering, as announced in the introduction.

(4.1) Before starting the proof we will need a few preliminaries. From now on we denote by $\mathrm{X}$ a compact complex surface; we assume given a direct sum decomposition $\Omega_{\mathrm{X}}^{1} \cong \mathrm{L} \oplus \mathrm{M}$. By lemma 3.1 (or by [B-B]) the Chern class $c_{1}(\mathrm{~L}) \in \mathrm{H}^{1}\left(\mathrm{X}, \Omega_{\mathrm{X}}^{1}\right)$ belongs to the subspace $\mathrm{H}^{1}(\mathrm{X}, \mathrm{L})$, and similarly for $\mathrm{M}$. As a consequence, we get:

(4.2) We have $\mathrm{L}^{2}=\mathrm{M}^{2}=0$, and therefore $c_{1}^{2}(\mathrm{X})=2 \mathrm{~L} \cdot \mathrm{M}=2 c_{2}(\mathrm{X})$.

The following consequence is less obvious.

Proposition 4.3 - Let $\mathrm{C}$ be a smooth rational curve in $\mathrm{X}$. Then $\mathrm{C}^{2} \geq 0$.

Proof: Put $\mathrm{C}^{2}=-d$ and assume $d>0$. Since $\mathrm{H}^{1}\left(\mathrm{C}, \mathcal{O}_{\mathrm{C}}(d+2)\right)=0$, the exact sequence

$$
0 \rightarrow \mathcal{O}_{\mathrm{C}}(d) \longrightarrow \Omega_{\mathrm{X} \mid \mathrm{C}}^{1} \longrightarrow \Omega_{\mathrm{C}}^{1} \rightarrow 0
$$

splits, giving an isomorphism $\Omega_{\mathrm{X} \mid \mathrm{C}}^{1} \cong \mathcal{O}_{\mathrm{C}}(d) \oplus \mathcal{O}_{\mathrm{C}}(-2)$. Thus one of the line bundles $\mathrm{L}$ or $\mathrm{M}$, say $\mathrm{L}$, satisfies $\mathrm{L}_{\mid \mathrm{C}} \cong \mathcal{O}_{\mathrm{C}}(d)$. Consider the commutative diagram 


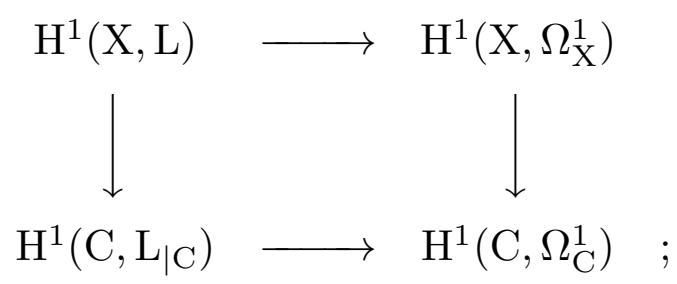

since $d>0$ we have $\mathrm{H}^{1}\left(\mathrm{C}, \mathrm{L}_{\mid \mathrm{C}}\right)=0$; thus $c_{1}(\mathrm{~L})$ goes to 0 in $\mathrm{H}^{1}\left(\mathrm{C}, \Omega_{\mathrm{C}}^{1}\right)$, which means $d=0$, a contradiction.

(4.4) We shall come across situations where the vector bundle $\Omega_{\mathrm{X}}^{1}=\mathrm{L} \oplus \mathrm{M}$ appears as an extension

$$
0 \rightarrow \mathrm{P} \longrightarrow \Omega_{\mathrm{X}}^{1} \stackrel{p}{\longrightarrow} \mathrm{Q} \rightarrow 0
$$

of two line bundles $\mathrm{P}$ and $\mathrm{Q}$. In that case,

- either the restriction of $p$ to one of the direct summands of $\Omega_{\mathrm{X}}^{1}$, say $\mathrm{M}$, is surjective; then the exact sequence splits, $\mathrm{Q}$ is isomorphic to $\mathrm{M}$ and $\mathrm{P}$ to $\mathrm{L}$;

- or the restriction of $p$ to both $\mathrm{L}$ and $\mathrm{M}$ is not surjective; then there exists effective (non-zero) divisors $\mathrm{A}$ and $\mathrm{B}$, whose supports do not intersect, such that $\mathrm{L} \cong \mathrm{Q}(-\mathrm{A}), \mathrm{M} \cong \mathrm{Q}(-\mathrm{B})$ and $\mathrm{P} \cong \mathrm{Q}(-\mathrm{A}-\mathrm{B})$; the exact sequence does not split.

In particular, if $\operatorname{Hom}(\mathrm{P}, \mathrm{Q})=0$, the exact sequence splits.

(4.5) Finally we will need some classical facts about connections (see [E]). Let $p: \mathrm{M} \rightarrow \mathrm{B}$ be a smooth holomorphic map between complex manifolds, whose fibres are isomorphic to a fixed variety $\mathrm{F}$. A connection on $p$ is a splitting of the exact sequence

$$
0 \rightarrow p^{*} \Omega_{\mathrm{B}}^{1} \longrightarrow \Omega_{\mathrm{M}}^{1} \longrightarrow \Omega_{\mathrm{M} / \mathrm{B}}^{1} \rightarrow 0,
$$

that is a sub-bundle $\mathrm{L} \subset \Omega_{\mathrm{M}}^{1}$ mapping isomorphically onto $\Omega_{\mathrm{M} / \mathrm{B}}^{1}$; the connection is flat (or integrable) if $d \mathrm{~L} \subset \mathrm{L} \wedge \Omega_{\mathrm{M}}^{1}$ (this is automatic if $\mathrm{B}$ is a curve). In that case the group $\pi_{1}(\mathrm{~B})$ acts on $\mathrm{F}$ by complex automorphisms, and $\mathrm{M}$ is the fibre bundle on $\mathrm{B}$ with fibre $\mathrm{F}$ associated to the universal covering $\widetilde{\mathrm{B}} \rightarrow \mathrm{B}$, that is the quotient of $\widetilde{\mathrm{B}} \times \mathrm{F}$ by the group $\pi_{1}(\mathrm{~B})$ acting diagonally; the splitting $\Omega_{\mathrm{M}}^{1}=p^{*} \Omega_{\mathrm{B}}^{1} \oplus \mathrm{L}$ pulls back to the decomposition $\Omega_{\widetilde{\mathrm{B}} \times \mathrm{F}}^{1}=\Omega_{\widetilde{\mathrm{B}}}^{1} \oplus \Omega_{\mathrm{F}}^{1}$.

\section{Proof of theorem $\mathrm{C}$}

\section{(5.1) Kodaira dimension 2}

If $\kappa(\mathrm{X})=2$, the canonical bundle $\mathrm{K}_{\mathrm{X}}$ is ample by Prop. 4.3. The Aubin-CalabiYau theorem implies that $\mathrm{X}$ admits a Kähler-Einstein metric; we can therefore apply Theorem A. 


\section{(5.2) Kodaira dimension 1}

If $\kappa(\mathrm{X})=1, \mathrm{X}$ admits an elliptic fibration $p: \mathrm{X} \rightarrow \mathrm{B}$. By 4.2 we have $c_{2}(\mathrm{X})=0$; this implies that the only singular fibres of $p$ are multiples of smooth elliptic curves (see [B1], VI.4 and VI.5). For $b \in \mathrm{B}$, we write $p^{*}[b]=m(b) \mathrm{F}_{b}$, where $\mathrm{F}_{b}$ is a smooth elliptic curve; we have $m(b) \geq 1$ and $m(b)=1$ except for finitely many points. Put $\Delta=\sum_{b}(m(b)-1) \mathrm{F}_{b}$. We have an exact sequence

$$
0 \rightarrow p^{*} \Omega_{\mathrm{B}}^{1}(\Delta) \longrightarrow \Omega_{\mathrm{X}}^{1} \longrightarrow \omega_{\mathrm{X} / \mathrm{B}} \rightarrow 0
$$

where $\omega_{\mathrm{X} / \mathrm{B}}$ is the relative dualizing line bundle. Since $\chi\left(\mathcal{O}_{\mathrm{X}}\right)=0$ by RiemannRoch, we deduce from [B-P-V], V.12.2 and III.18.2, that $\omega_{\mathrm{X} / \mathrm{B}}$ is a torsion line bundle. Since $\mathrm{K}_{\mathrm{X}}=p^{*} \Omega_{\mathrm{B}}^{1}(\Delta) \otimes \omega_{\mathrm{X} / \mathrm{B}}$, the hypothesis $\kappa(\mathrm{X})=1$ implies $\operatorname{Hom}\left(p^{*} \Omega_{\mathrm{B}}^{1}(\Delta), \omega_{\mathrm{X} / \mathrm{B}}\right)=0$, hence the exact sequence (5.3) splits by 4.4 .

Let $\rho: \widetilde{\mathrm{B}} \rightarrow \mathrm{B}$ be the orbifold universal covering of $(\mathrm{B}, m)$ : this is a ramified Galois covering, with $\widetilde{\mathrm{B}}$ simply-connected, such that the stabilizer of a point $\tilde{b} \in \widetilde{\mathrm{B}}$ is a cyclic group of order $m(\rho(\tilde{b}))$ (see for instance [K-O], lemma 6.1; note that because of the hypothesis $\kappa(\mathrm{X})=1$ and the formula for $\mathrm{K}_{\mathrm{X}}$, there are at least 3 multiple fibers if $\mathrm{B}$ is of genus 0 ). Let $\widetilde{\mathrm{X}}$ be the normalization of $\mathrm{X} \times{ }_{\mathrm{B}} \widetilde{\mathrm{B}}$. We have a commutative diagram

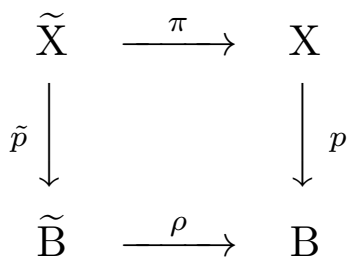

where $\tilde{p}$ is smooth and $\pi$ is étale ([B1], VI. $\left.7^{\prime}\right)$. The exact sequence

$$
0 \rightarrow \tilde{p}^{*} \Omega_{\widetilde{\mathrm{B}}}^{1} \longrightarrow \Omega_{\widetilde{\mathrm{X}}}^{1} \longrightarrow \Omega_{\widetilde{\mathrm{X}} / \widetilde{\mathrm{B}}}^{1} \rightarrow 0
$$

coincides with the pull back under $\pi$ of the exact sequence (5.3); therefore $p$ admits an integrable connection, given by the subbundle $\pi^{*} \mathrm{M}$ of $\Omega_{\widetilde{\mathrm{X}}}^{1}$. The result follows from 4.5 and 1.2 .

(5.4) Kodaira dimension 0

Assume $\kappa(\mathrm{X})=0$. By 4.2 and the classification of surfaces, $\mathrm{X}$ is either a complex torus, a bielliptic surface, or a Kodaira surface. Complex tori and bielliptic surfaces fall into case $a$ ) of the theorem (a bielliptic surface is the quotient of a product $\mathrm{E} \times \mathrm{F}$ of elliptic curves by a finite abelian group acting diagonally).

A primary Kodaira surface has trivial canonical bundle and admits a smooth elliptic fibration $p: \mathrm{X} \rightarrow \mathrm{B}$. Thus the exact sequence (4.2) realizes $\Omega_{\mathrm{X}}^{1}$ as an 
extension of $\mathcal{O}_{\mathrm{X}}$ by $\mathcal{O}_{\mathrm{X}}$. Since $h^{1,0}(\mathrm{X})=1$, this extension is non-trivial, and it follows from 4.4 that $\Omega_{\mathrm{X}}^{1}$ does not split.

A secondary Kodaira surface admits a primary Kodaira surface as a finite étale cover, hence its tangent bundle cannot split either.

\section{(5.5) Ruled surfaces}

We consider the case when $\mathrm{X}$ is algebraic and $\kappa(\mathrm{X})=-\infty$. By 4.2 and $4.3, \mathrm{X}$ is a geometrically ruled surface, that is a projective bundle $p: \mathrm{X} \rightarrow \mathrm{B}$ over a curve. We again consider the exact sequence

$$
0 \rightarrow p^{*} \Omega_{\mathrm{B}}^{1} \longrightarrow \Omega_{\mathrm{X}}^{1} \longrightarrow \Omega_{\mathrm{X} / \mathrm{B}}^{1} \rightarrow 0
$$

since $\Omega_{\mathrm{X} / \mathrm{B}}^{1}$ has negative degree on the fibres, we have $\operatorname{Hom}\left(p^{*} \Omega_{\mathrm{B}}^{1}, \Omega_{\mathrm{X} / \mathrm{B}}^{1}\right)=0$, hence by 4.4 the above exact sequence splits: one of the direct summands of $\Omega_{\mathrm{X}}^{1}$ defines an integrable connection for $p$. The result follows then from 4.5.

(5.6) Inoue surfaces

We now assume that $\mathrm{X}$ is not algebraic and $\kappa(\mathrm{X})=-\infty$, so that $\mathrm{X}$ is what is usually called a surface of type $\mathrm{VII}_{0}$. These surfaces have $b_{1}=h^{0,1}=1$ and therefore $c_{1}^{2}+c_{2}=12 \chi\left(\mathcal{O}_{\mathrm{X}}\right)=0$; in our case this gives $c_{1}^{2}=c_{2}=0$ in view of 4.2, and finally $b_{2}=0$. Moreover we have $\mathrm{H}^{0}\left(\mathrm{X}, \Omega_{\mathrm{X}}^{1} \otimes \mathrm{L}^{-1}\right) \neq 0$. The surfaces with these properties have been completely classified by Inoue [I]: they are either Hopf surfaces, or belong to three classes of surfaces constructed by Inoue (loc. cit.).

We first consider the Inoue surfaces. The surfaces $S_{M}$ of the first class are quotients of $\mathbf{H} \times \mathbf{C}$ by a group acting diagonally, hence they fall into case $a$ ) of the theorem.

The surfaces $\mathrm{S}_{\mathrm{N}, p, q, r ; t}^{(+)}$of the second class are quotients of $\mathbf{H} \times \mathbf{C}$ by a group which does not act diagonally. This action leaves invariant the vector field $\partial / \partial z$ on $\mathbf{C}$, which therefore descends to a non-vanishing vector field $v$ on $\mathbf{X}$. This gives rise to an exact sequence

$$
0 \rightarrow \mathrm{K}_{\mathrm{X}} \stackrel{i(v)}{\longrightarrow} \Omega_{\mathrm{X}}^{1} \stackrel{i(v)}{\longrightarrow} \mathcal{O}_{\mathrm{X}} \rightarrow 0
$$

which does not split since $h^{1,0}(\mathrm{X})=0$. We have $\mathrm{H}^{0}\left(\mathrm{X}, \mathrm{K}_{\mathrm{X}}^{-1}\right)=0$, for instance because $\mathrm{X}$ contains no curves; we infer from 4.4 that $\Omega_{\mathrm{X}}^{1}$ does not split.

The surfaces $\mathrm{S}_{\mathrm{N}, p, q, r}^{(-)}$of the third class are quotients of certain surfaces of the second class by a fixed point free involution; therefore their tangent bundle does not split either.

\section{(5.7) Primary Hopf surfaces}

It remains to consider the class of Hopf surfaces, which are by definition the surfaces of class $\mathrm{VII}_{0}$ whose universal covering space is $\mathbf{W}:=\mathbf{C}^{2}-\{0\}$. We 
consider first the primary Hopf surfaces, which are quotients of $\mathbf{W}$ by the infinite cyclic group generated by an automorphism $\mathrm{T}$ of $\mathbf{W}$. According to [Ko], $\S 10$, there are two cases to consider:

a) $\mathrm{T}(x, y)=(\alpha x, \beta y)$ for some complex numbers $\alpha, \beta$ with $0<|\alpha| \leq|\beta|<1$;

b) $\mathrm{T}(x, y)=\left(\alpha^{m} x+\lambda y^{m}, \alpha y\right)$ for some positive integer $m$ and non-zero complex numbers $\alpha, \lambda$ with $|\alpha|<1$.

As in 2.1, we denote by $\mathrm{L}_{\theta}$, for $\theta \in \mathbf{C}$, the flat line bundle associated to the character of $\pi_{1}(\mathrm{X})$ mapping $\mathrm{T}$ to $\theta$. In case $a$ ) we find $\Omega_{\mathrm{X}}^{1}=\mathrm{L}_{\alpha}^{-1} \oplus \mathrm{L}_{\beta}^{-1}$, so the tangent bundle splits.

Let us consider case $b$ ). The form $d y$ on $\mathbf{W}$ satisfies $\mathrm{T}^{*} d y=\alpha d y$, hence descends to a form $\overline{d y}$ in $\mathrm{H}^{0}\left(\mathrm{X}, \Omega_{\mathrm{X}}^{1} \otimes \mathrm{L}_{\alpha}\right)$; similarly the function $y$ descends to a non-zero section of $\mathrm{L}_{\alpha}$. We have an exact sequence

$$
0 \rightarrow \mathrm{L}_{\alpha}^{-1} \stackrel{\overline{d y}}{\longrightarrow} \Omega_{\mathrm{X}}^{1} \longrightarrow \mathrm{L}_{\alpha}^{-m} \rightarrow 0 .
$$

Since $\mathrm{L}_{\alpha}$ has a non-zero section, the space $\operatorname{Hom}\left(\mathrm{L}_{\alpha}^{-1}, \mathrm{~L}_{\alpha}^{-m}\right)$ is zero for $m>1$. Hence if $\Omega_{\mathrm{X}}^{1}$ splits, we deduce from 4.4 that the exact sequence splits. This means that there exists a form $\bar{\omega} \in \mathrm{H}^{0}\left(\mathrm{X}, \Omega_{\mathrm{X}}^{1} \otimes \mathrm{L}_{\alpha}^{m}\right)$ such that $\bar{\omega} \wedge \overline{d y} \neq 0$. Then $\bar{\omega} \wedge \overline{d y}$ is a generator of the trivial line bundle $\mathrm{K}_{\mathrm{X}} \otimes \mathrm{L}_{\alpha}^{m+1}$, hence pulls back to $c d x \wedge d y$ on $\mathbf{W}$, for some constant $c \neq 0$. Therefore the pull back $\omega$ of $\bar{\omega}$ to $\mathbf{W}$ is of the form $c d x+f(x, y) d y$ for some holomorphic function $f$ on $\mathbf{C}^{2}$. The flat line bundle $\mathrm{L}_{\alpha}^{m}$ carries a flat holomorphic connection $\nabla$; the 2 -form $\nabla \bar{\omega}$, which is a global section of $\mathrm{K}_{\mathrm{X}} \otimes \mathrm{L}_{\alpha}^{m} \cong \mathrm{L}_{\alpha}^{-1}$, is zero. This implies $d \omega=0$, so the function $f(x, y)$ is independent of $x$; let us write it $f(y)$. Now the condition $\mathrm{T}^{*} \omega=\alpha^{m} \omega$ reads $\alpha f(\alpha y)+c \lambda m y^{m-1}=\alpha^{m} f(y)$. Differentiating $m$ times we find $f^{(m)}=0$, then differentiating $m-1$ times leads to a contradiction.

\section{(5.8) Secondary Hopf surfaces}

A secondary Hopf surface $\mathrm{X}$ is the quotient of $\mathbf{W}$ by a group $\Gamma$ acting freely, containing a central, finite index subgroup generated by an automorphism $\mathrm{T}$ of the above type. We assume that $\Omega_{\mathrm{X}}^{1}$ splits. The primary Hopf surface $\mathrm{Y}=\mathbf{W} / \mathrm{T}^{\mathbf{Z}}$ is a finite étale cover of $\mathrm{X}$, so $\Omega_{\mathrm{Y}}^{1}$ also splits; it follows from 5.7 that $\mathrm{T}$ is of type $a$ ), and that $\Gamma$ does not contain any transformation of type $b$ ). According to $[\mathrm{Ka}]$, $\S 3$, this implies that after an appropriate change of coordinates, the group $\Gamma$ acts linearly on $\mathbf{C}^{2}$.

We claim that $\Gamma$ is contained in a maximal torus of $\mathbf{G L}(2, \mathbf{C})$. This is clear if $\alpha \neq \beta$, because $\mathrm{T}$ is central in $\Gamma$. If $\alpha=\beta$, the direct sum decomposition of $\Omega_{\mathrm{X}}^{1}$ pulls back to a decomposition $\Omega_{\mathrm{Y}}^{1}=\mathrm{L}_{\alpha}^{-1} \oplus \mathrm{L}_{\alpha}^{-1}$ (5.7), which for an appropriate choice of coordinates comes from the decomposition $\Omega_{\mathbf{W}}^{1}=\mathcal{O}_{\mathbf{W}} d x \oplus \mathcal{O}_{\mathbf{W}} d y$. Since $\Gamma$ must preserve this decomposition, it is contained in the diagonal torus. 
Thus we may identify $\Gamma$ with a subgroup of $\left(\mathbf{C}^{*}\right)^{2}$; since it acts freely on $\mathbf{W}$, the first projection $\Gamma \rightarrow \mathbf{C}^{*}$ is injective. Therefore the torsion subgroup of $\Gamma$ is cyclic, and we are in case $b$ ) of the theorem.

\section{REFERENCES}

[B1] A. Beauville: Surfaces algébriques complexes. Astérisque 54 (1978).

[B2] A. Beauville: Variétés kählériennes dont la première classe de Chern est nulle. J. of Diff. Geometry 18, 755-782 (1983).

[B-B] P. Baum, R. Вотт: On the zeros of meromorphic vector-fields. Essays on Topology and Related Topics, 29-47; Springer, New York (1970).

[B-P-V] W. Barth, C. Peters, A. Van de Ven: Compact complex surfaces. Ergebnisse der Math., Springer-Verlag (1984).

[E] C. Ehresmann: Les connexions infinitésimales dans un espace fibré différentiable. Colloque de topologie, Bruxelles (1950), 29-55. G. Thone, Liège (1951).

[I] M. Inoue: On surfaces of class VII ${ }_{0}$. Invent. math. 24, 269-310 (1974).

[Ka] M. Kato: Topology of Hopf surfaces. J. Math. Soc. Japan 27, 222-238 (1975).

[K] S. Kobayashi: First Chern class and holomorphic tensor fields. Nagoya Math. J. 77, 5-11 (1980).

[K-O] S. Kobayashi, T. Ochiai: Holomorphic structures modeled after hyperquadrics. Tôhoku Math. J. 34, 587-629 (1982).

[Ko] K. Kodaira: On the structure of compact complex analytic surfaces II. Amer. J. of Math. 88, 682-721 (1966).

[S] C. Simpson: Constructing variations of Hodge structure using Yang-Mills theory and applications to uniformization. J. Amer. Math. Soc. 1, 867-918 (1988).

[Y] S.-T. YAU: A splitting theorem and an algebraic geometric characterization of locally hermitian symmetric spaces. Comm. in Analysis and Geometry 1, 473-486 (1993). 